\title{
H)axtin Dpitgen
}

Aristarchus sive de contemptu linguae

\author{
Teutonicae
}

und

Buch von der Deutichen Poeterey.

Gerausgegeben

von

Dr. Georg Wittowati.

此ipziy,

Derlag von Deit \& Comp.

1888. 

Ģerrn

Geh. Gofrat Profeffor

\title{
$\mathfrak{f r i e d r i c h ~} \mathfrak{o} \mathfrak{a} \mathfrak{r} \mathfrak{a} \mathfrak{e}$
}

\author{
in Janlbarer Verehrung
}

gewiomet. 
